\title{
AN INVERSE FUNCTION THEOREM FOR FREE GROUPS
}

\author{
JOAN S. BIRMAN ${ }^{1}$
}

\begin{abstract}
Let $F_{n}$ be a free group of rank $n$ with free basis $x_{1}, \cdots, x_{n}$. Let $\left\{y_{1}, \cdots, y_{k}\right\}$ be a set of $k \leqq n$ elements of $F_{n}$, where each $y_{i}$ is represented by a word $Y_{i}\left(x_{1}, \cdots, x_{n}\right)$ in the generators $x_{j}$. Let $\partial y_{i} / \partial x_{j}$ denote the free derivative of $y_{i}$ with respect to $x_{j}$, and let $J_{k n}=\left\|\partial y_{i} / \partial x_{j}\right\|$ denote the $k \times n$ Jacobian matrix. THEOREM. If $k=n$, the set $\left\{y_{1}, \cdots, y_{n}\right\}$ generates $F_{n}$ if and only if $J_{n n}$ has a right inverse. If $k<n$, the set $\left\{y_{1}, \cdots, y_{k}\right\}$ may be extended to a set of elements which generate $F_{n}$ only if $J_{k n}$ has a right inverse. Several applications are given.
\end{abstract}

Let $Z$ denote the ring of rational integers, and let $Z F_{n}$ denote the integral group ring of a free group $F_{n}$ which has the free basis $x_{1}, \cdots, x_{n}$. Let $\partial / \partial x_{j}: Z F_{n} \rightarrow Z F_{n}$ denote the $j$ th free partial derivative, in the sense of R. H. Fox [3]. The mapping $\partial / \partial x_{j}$ is defined as follows: If $w \in F_{n}$ is represented by the word $x_{\mu_{1}}^{\varepsilon_{1}} \cdots x_{\mu_{r}}^{\varepsilon_{r}}$, where $\varepsilon_{i}= \pm 1$ and $\mu_{i}=1, \cdots, n$, then $\partial w / \partial x_{j} \in Z F_{n}$ is defined by:

$$
\frac{\partial w}{\partial x_{j}}=\sum_{k=1}^{n} \delta_{j, \mu_{k}} \varepsilon_{k} x_{\mu_{2}}^{\varepsilon_{1}} \cdots x_{\mu_{k-1}}^{\varepsilon_{k-1}} x_{\mu_{k}}^{\left(\varepsilon_{k}-1\right) / 2}
$$

where $\delta$ means the Kronecker symbol. More generally, if $u=\sum_{i=1}^{t} c_{i} w_{i}$, $c_{i} \in Z, w_{i} \in F_{n}$, we define

$$
\frac{\partial u}{\partial x_{j}}=\sum_{i=1}^{t} c_{i} \frac{\partial w_{i}}{\partial x_{j}}
$$

It is easy to show that this definition is independent of the choice representatives of the elements $w_{i} \in F_{n}$.

There are known analogues between the "free" calculus of polynomials in the noncommuting indeterminates $x_{1}, \cdots, x_{n}$ and the "ordinary" calculus of polynomials in commuting indeterminates, such as the existence of Taylor series [3]. However, it is worth noting that if $\alpha$ is the

Received by the editors November 30, 1972 and, in revised form, February 26, 1973. AMS (MOS) subject classifications (1970). Primary 55A05, 20E99; Secondary 20F55. Key words and phrases. Free calculus, primitive elements, free basis, invertible matrices.

${ }^{1}$ This work was supported in part by NSF Grant GP-34324X.

(c) American Mathematical Society 1973 
abelianizing homomorphism acting on $F_{n}$, and if $\alpha_{*}$ is the induced homomorphism from $Z F_{n} \rightarrow Z F_{n}^{\alpha}$, then the image under $\alpha_{*}$ of the free partial derivative of $u$, as defined by (2), is not the ordinary partial derivative of $\alpha_{*}(u)$. Thus the free calculus and the ordinary calculus appear to be distinct theories. The purpose of this note is to point out a new analogue between the free calculus and the ordinary calculus, an "inverse function theorem" for free groups.

INVERSE FUNCTION THEOREM. Let $\left\{y_{1}, \cdots, y_{k}\right\}$ be a set of $k \leqq n$ elements of $F_{n}$. Let $J_{k n}$ denote the $k \times n$ "Jacobian" matrix $\left\|\partial y_{i} \mid \partial x_{j}\right\|$.

(i) If $k=n$, a necessary and sufficient condition for $\left\{y_{1}, \cdots, y_{n}\right\}$ to be a generating set for $F_{n}$ is that $J_{n n}$ have a right inverse.

(ii) If $k<n$, a necessary condition for $\left\{y_{1}, \cdots, y_{k}\right\}$ to extend to a generating set $\left\{y_{1}, \cdots, y_{n}\right\}$ is that $J_{k n}$ have a right inverse.

Proof. We first establish the sufficiency of the condition (i). ${ }^{2}$ Suppose that $B=\left\|\beta_{i j}\right\|$ is a right inverse of $J_{n n}$. By a theorem of M. S. Montgomery [6], the matrix $B$ is also a left inverse of $J_{n n}$. Hence

$$
\sum_{s=1}^{n} \beta_{i s}\left(\frac{\partial y_{s}}{\partial x_{j}}\right)=\delta_{i j} \quad(i, j=1, \cdots, n) .
$$

Multiplying both sides of (3) by $x_{j}-1$, and summing over $j$, we obtain

$$
\sum_{s=1}^{n} \beta_{i s} \sum_{j=1}^{n} \frac{\partial y_{s}}{\partial x_{j}}\left(x_{j}-1\right)=x_{i}-1 \quad(i=1, \cdots, n) .
$$

By the "fundamental theorem" of free calculus [3]:

Hence

$$
\sum_{j=1}^{n} \frac{\partial y_{s}}{\partial x_{j}}\left(x_{j}-1\right)=y_{s}-1 \quad(s=1, \cdots, n) .
$$

$$
\sum_{s=1}^{n} \beta_{i s}\left(y_{s}-1\right)=x_{i}-1 \quad(i=1, \cdots, n) .
$$

Now let $H$ be the subgroup of $F_{n}$ generated by $y_{1}, \cdots, y_{n}$ and let $I_{H}$ be the ideal of $Z F_{n}$ generated by $y_{1}-1, \cdots, y_{n}-1$. According to equation (6), the ring elements $x_{i}-1$ belong to $I_{H}$ for each $i=1, \cdots, n$. But then, by Lemma 4.1 of [2], it follows that $x_{i} \in H$ for each $i=1, \cdots, n$. Hence $H$ coincides with $F_{n}$, and our result is established.

Necessity may be established by noting that if $\left\{y_{1}, \cdots, y_{k}\right\}$ extends to a basis $\left\{y_{1}, \cdots, y_{n}\right\}$, then we may write each $x_{i}$ as a word $X_{i}\left(y_{1}, \cdots, y_{n}\right)$,

${ }^{2}$ The very brief proof of sufficiency given here was suggested by the referee. It replaces a longer and more computational proof in an earlier version of this paper. The author wishes to thank the referee for his constructive suggestions. 
in the generators $y_{j}$. Moreover, if $y_{i}$ is represented by the word $Y_{i}\left(x_{1}, \cdots, x_{n}\right)$, then we will have

$$
Y_{i}\left(X_{1}\left(y_{1}, \cdots, y_{n}\right), \cdots, X_{n}\left(y_{1}, \cdots, y_{n}\right)\right)=y_{i} \quad(i=1, \cdots, n) .
$$

The chain rule (see [3]) applied to (7) gives

$$
\sum_{s=1}^{n}\left(\frac{\partial Y_{i}\left(x_{1}, \cdots, x_{n}\right)}{\partial x_{s}}\right)\left(\frac{\partial X_{s}\left(y_{1}, \cdots, y_{n}\right)}{\partial y_{j}}\right)=\delta_{i j} \quad(i, j=1, \cdots, n) .
$$

Hence the $n \times n$ matrix $J_{n n}$ has a right inverse, $J_{n n}^{*}=\left\|\partial x_{i} / \partial y_{j}\right\|$. Thus condition (i) is seen to be sufficient. Also the $k \times n$ submatrix $J_{k n}$ formed by the first $k$ rows of $J_{n n}$ has a right inverse, namely the submatrix $J_{n k}^{*}$ of $J_{n n}^{*}$ formed by the first $k$ columns of $J_{n n}^{*}$. This proves (ii).

As an application, we will use the inverse function theorem to give a new proof of a classical theorem of J. Nielsen. This application was suggested by the referee. The proof below is a modification of his proof.

Corollary 1 (J. Nielsen, see [5]). Any set of $n$ elements which generate a free group of rank $n$ are a set of free generators.

Proof. Suppose that $\left\{y_{1}, \cdots, y_{n}\right\}$ generate $F_{n}$, and suppose also that $\left\{y_{1}, \cdots, y_{n}\right\}$ satisfy the relation

$$
r\left(y_{1}, \cdots, y_{n}\right)=1 \text {, }
$$

where we assume that $r\left(y_{1}, \cdots, y_{n}\right)$ is freely reduced as a word in the $y_{j}$ 's. By the chain rule it follows that

$$
\sum_{\partial=1}^{n} \frac{\partial r}{\partial y_{j}} \frac{\partial y_{j}}{\partial x_{i}}=0, \quad i=1, \cdots, n .
$$

Setting $r=\left(\partial r / \partial y_{1}, \cdots, \partial r / \partial y_{n}\right)$, we may rewrite (10) in the form

$$
\boldsymbol{r} J_{n n}=\boldsymbol{o} .
$$

By the inverse function theorem, the matrix $J_{n n}$ has a right inverse, say $B$. Multiplying both sides of (11) by $B$ we obtain

$$
\boldsymbol{r} J_{n n} B=\boldsymbol{r} I=\boldsymbol{r}=\boldsymbol{o},
$$

hence $\partial r / \partial y_{j}=0$ for each $j=1, \cdots, n$. But then it follows that $r$ does not involve the letter $y_{j}$, since no cancellations are possible in (1) if a word is freely reduced. It then follows that $r$ must be the trivial relator. This completes the proof of Corollary 1.

We observe that our theorem may be applied both ways. Consider first the case $k=n$. An algorithm for deciding whether a set of $n$ elements 
in a free group $F_{n}$ are a basis was discovered by J. Nielsen (see Chapter 2 of [5]), and Nielsen's algorithm translates into a straightforward algorithm for expressing a Jacobian matrix as a product of elementary invertible matrices over $Z F_{n}{ }^{3}$ On the other hand, it is known [7] that the ring $Z F_{n}$ can be embedded in a skew field $K$. Since a procedure exists for finding inverses of invertible matrices over skew fields (see Chapter IV of [1]), and since such inverses are unique, one may decide whether a Jacobian matrix is invertible over $Z F_{n}$ by computing its inverse over $K$, and seeing whether in fact the entries are in $Z F_{n}$. This yields a new test to decide if $y_{1}, \cdots, y_{n}$ are a basis. This procedure is not however, a practical alternative to Nielsen's relatively simple algorithm; the elementary invertible matrices which are obtained by the method in [1] are almost certainly not in $Z F_{n}$, even when their product is in $Z F_{n}$, so that this procedure is unnecessarily complex. Our theorem does, however, yield a very simple necessary condition which a set $\left\{y_{1}, \cdots, y_{n}\right\}$ must survive if it is a basis:

COROllary 2. Let $J_{n n}^{\alpha}$ denote the image of $J_{n n}$ under the abelianizing homomorphism $\alpha_{*}$ acting on $Z F_{n}$. Then $\left\{y_{1}, \cdots, y_{n}\right\}$ is a basis for $F_{n}$ only if $\operatorname{det} J_{n n}^{\alpha}$ is a unit in $Z F_{n}^{\alpha}$.

Proof. By Theorem 1, p. 59, of [4], a square matrix over a commutative ring with 1 is invertible if and only if its determinant is a unit.

To see that the condition in Corollary 2 is not sufficient, let $n=2$ and consider the elements

$$
\begin{aligned}
& y_{1}=x_{1}, \\
& y_{2}=x_{2} x_{1} x_{2} x_{1}^{-1} x_{2}^{-1} x_{1}^{2} x_{2}^{2} x_{1}^{-2} x_{2}^{-1} x_{1} x_{2}^{-1} x_{1}^{-1} x_{2}^{2} x_{1}^{2} x_{2}^{-2} x_{1}^{-2} .
\end{aligned}
$$

A simple calculation shows that $\operatorname{det}\left\|\partial y_{i} / \partial x_{j}\right\|^{\alpha}=1$, yet $y_{1}$ and $y_{2}$ are not primitive, by the test given in Corollary N4, p. 169, of [5].

The more difficult question of deciding whether a set of $k<n$ elements in a free group are primitive was solved by J. H. C. Whitehead [9], [10] and by E. Rapaport [8], and the inverse function theorem may be applied to yield an analogous algorithm for deciding when a $k \times n$ Jacobian matrix over $Z F_{n}$ has a right inverse. Once again, the Jacobian matrices corresponding to Whitehead transformations are a very pleasant set of elementary invertible matrices.

\footnotetext{
${ }^{3}$ Note that the mapping which we have defined from Aut $F_{n}$ to the ring of invertible matrices over $Z F_{n}$ is a crossed homomorphism. That is, if $\alpha$ and $\beta$ are automorphisms of $F_{n}$ which have Jacobian matrices $\left\|a_{i j}\right\|$ and $\left\|b_{i j}\right\|$ respectively, then the Jacobian matrix corresponding to $\alpha \beta$ is the product $\left\|a_{i j}\right\|\left\|b_{i j}\right\|_{\alpha}$, where $\left\|b_{i j}\right\|_{\alpha}$ denotes the Jacobian matrix of $\beta$ with respect to the transformed basis $\alpha\left(x_{1}\right), \cdots, \alpha\left(x_{n}\right)$. Thus, in order to apply Nielsen's algorithm, one must repeatedly change basis.
} 


\section{REFERENCES}

1. E. Artin, Geometric algebra, Interscience Tracts in Pure and Appl. Math., no. 3, Interscience, New York, 1957. MR 18, 553.

2. D. Cohen, Groups of cohomological dimension one, Lecture Notes in Math., vol. 245, Springer-Verlag, Berlin and New York, 1972.

3. R. H. Fox, Free differential calculus. I. Derivation in the free group ring, Ann. of Math. (2) 57 (1953), 547-560. MR 14, 843.

4. N. Jacobson, Lectures in abstract algebra. I. Basic concepts, Van Nostrand, Princeton, N.J., 1951. MR 12, 794.

5. W. Magnus, A. Karrass and D. Solitar, Combinatorial group theory. Presentations of groups in terms of generators and relations, Pure and Appl. Math. vol. 13, Interscience, New York, 1966. MR 34 \#7617.

6. M. S. Montgomery, Left and right inverses in group algebras, Bull. Amer. Math. Soc. 75 (1969), 539-540. MR 39 \#327.

7. B. Neumann, On ordered division rings, Trans. Amer. Math. Soc. 66 (1949), 202-252. MR 11, 311.

8. E. S. Rapaport, On free groups and their automorphisms, Acta Math. 99 (1958), 139-163. MR 24 \#A1302.

9. J. H. C. Whitehead, On certain sets of elements in a free group, Proc. London Math. Soc. 41 (1936), 48-56.

10. - On equivalent sets of elements in a free group, Ann. of Math. 37 (1936), 782-800.

Department of Mathematics, Stevens Institute of Technology, Hoboken, NEW JERSEY 07030

Current address: Department of Mathematics, Columbia University, New York, New York 10027 